\title{
ANÁLISIS DE LAS CAPTURAS DE TIBURONES Y RAYAS EN LAS PESQUERÍAS ARTESANALES DE TÁRCOLES, PACÍFICO CENTRAL DE COSTA RICA
}

\author{
Andrés López Garro ${ }^{1 *}$, Rándall Arauz Vargas ${ }^{1}$, Ilena Zanella ${ }^{1}$ y Loic Le Foulgo ${ }^{2}$
}

\begin{abstract}
RESUMEN
Se analizaron las capturas de tiburones y rayas látigo realizadas por pescadores artesanales de Tárcoles en el Pacífico de Costa Rica, desde febrero del 2006 hasta febrero del 2007. Se observaron 415 faenas de pesca (línea de fondo, trasmallo y línea rayera): 340 correspondieron a observaciones de descarga y $75(18.1 \%)$ a observaciones a bordo. Durante las descargas se analizaron 3.299 tiburones y rayas látigo: el tiburón mamón enano (Mustelus henlei) dominó en la captura total (63.7\%), seguido por el tiburón mamón común (Mustelus lunulatus) (16.7\%), el tiburón picudo común (Rhizoprionodon longurio) (6.9\%) y el tiburón martillo común (Sphyrna lewini) (6.1\%). A su vez, la raya látigo (D. longa) y el tiburón picudo fucsia (Nasolamia velox) representaron un $3.5 \%$ y $2.6 \%$ de la captura total, respectivamente. En los 75 viajes a bordo se capturó un total de 6.458 peces, de los cuales solo el 4.4\% (240 individuos) fueron tiburones y rayas látigo. M. henlei fue nuevamente la especie más abundante (50.4\%), le siguieron $R$. longurio (21.7\%), D. longa (13.8), N. velox (5.8\%), S. lewini (5.8\%), y por último, M. lunulatus (2.5\%). Debido a la estacionalidad y abundancia de tiburones mamones en la zona de Herradura, se recomienda un cierre técnico en enero-febrero y setiembre-octubre, meses con las mayores capturas. También se recomienda la protección de la zona del Peñón, ya que es utilizada por la raya látigo y por los juveniles del tiburón martillo.
\end{abstract}

Palabras claves: Tiburones, rayas látigo, pesca artesanal, abundancia relativa, Costa Rica.

\begin{abstract}
From February 2006 to February 2007, we analyzed shark and rays captures by the artisanal fishermen from Tárcoles, in the Pacific of Costa Rica. We observed 415 fishing operations (bottom line, gillnet and ray line): 340 dock landing observations and 75 (18.1\%) on board observations. During the landing observations, we analyzed 3.299 sharks and rays: the brown smooth hound (Mustelus henlei) was the most common species (63.7\%), followed by the common smooth hound (Mustelus lunulatus) (16.7\%), the Pacific sharpnose shark (Rhizoprionodon longurio) (6.9\%) and the scalloped hammerhead shark (Sphyrna lewini) (6.1\%). The longtail stingray (Dasyatis longa) and the whitenose shark (Nasolamia velox) represented the $3.5 \%$ and $2.6 \%$ of the total catch, respectively. During 75 fishing trips, we analyzed 6.458 fish individuals, of them only 4.4\% (240 individuals) were sharks and longtail stingrays. Among these, $M$. henlei was also the most abundant species $(50.4 \%)$, followed by R. longurio (21.7\%), D. longa (13.8\%), N. velox (5.8\%), S. lewini (5.8\%), and M. lunulatus (2.5\%). Considering the seasonality and abundance of smooth hound sharks in Herradura, we recommend a technical closure during the higher catch months (January-February and September-October). Also, we recommend protecting the Peñon, which is inhabited by adults of longtail stingray and juvenile scalloped hammerhead sharks.
\end{abstract}

Keywords: Sharks, longtail stingrays, artisanal fishery, relative abundance, Costa Rica.

1 PRETOMA: Programa de Restauración de Tortugas Marinas. San José, Costa Rica. Email alopez@, misiontiburon.org*,rarauz@pretoma.org e izanella@misiontiburon.org

2 Université de La Rochelle. La Rochelle, France. Email loiclefoulgoc@yahoo.fr

Recibido 29-V-2009

Aceptado 30-IX-2009

DOI: http://dx.doi.org/10.15359/revmar.1.8

Rev. Mar. y Cost. ISSN 1659-455X. Vol. 1. 145-157, Diciembre 2009. 


\section{INTRODUCCIÓN}

Con una extensión marina de más de $500.000 \mathrm{~km}^{2}$, las aguas del Pacífico costarricense representan la principal fuente alimenticia y económica para diferentes comunidades, ya que más del $98 \%$ de los productos pesqueros que se comercializan en Costa Rica provienen de esta región (González et al. 1993). De hecho, con el paso del tiempo se han desarrollado importantes comunidades pesqueras, donde la mayor parte de los habitantes dependen de esta actividad para subsistir. Tal es el caso de la comunidad de Tárcoles en el Pacífico Central de Costa Rica, donde la pesca artesanal representa el mayor sostén económico (Coope SoliDar R.L., 2005).

En las pesquerías artesanales de Tárcoles, las especies objetivo o de mayor valor económico son principalmente los pargos (Lutjanidae) y los róbalos (Centropomidae), sin embargo, se capturan especies de tiburones y rayas de manera incidental (Villareal, 2001).

La disminución de las especies costeras objetivo hace que actualmente los tiburones sean más comunes en las capturas pesqueras (INRECOSMAR, 1999). El problema es que los tiburones, debido a sus características biológicas, no son capaces de soportar fuertes presiones pesqueras por largo tiempo, como consecuencia muchas especies en este momento se encuentran amenazadas. Tales son los casos del tiburón martillo, el tiburón blanco y el tiburón zorro del Atlántico noroeste, cuyas poblaciones han disminuido un $75 \%$ en los últimos 15 años (Baum et al. 2003). Costa Rica no es la excepción a esta realidad, ya que estudios en la Zona Económica del Pacífico reportan una disminución en la abundancia relativa de tiburones de un 60\% entre 1991 y el 2001 (Arauz et al. 2004).

Este declive en las poblaciones de tiburones en el ámbito global está haciendo que se promuevan iniciativas que garanticen la conservación de las especies y hábitats más vulnerables, sin embargo, conocemos poco sobre las especies, biología y dinámica poblacional de los tiburones. Con el fin de mejorar el conocimiento sobre las especies de tiburón capturadas por las pesquerías artesanales del Pacífico de Costa Rica, la presente investigación analiza los datos de capturas de tiburones y rayas provenientes de las faenas costeras realizadas por los pescadores de Tárcoles en la parte externa del Golfo de Nicoya. A la vez, brinda información de la línea base sobre las especies de tiburones más abundantes en las pesquerías artesanales de Tárcoles.

\section{MATERIALES Y MÉTODOS}

Entre febrero del 2006 y febrero del 2007 se recolectaron los datos de las capturas de tiburones y rayas obtenidas por los pescadores artesanales de la comunidad de Tárcoles. Esta comunidad está ubicada a $37 \mathrm{~km}$ al sureste de Puntarenas, en el extremo oriental de la boca del Golfo de Nicoya (Figura 1). Al igual que en otras 


\section{Figura 1}

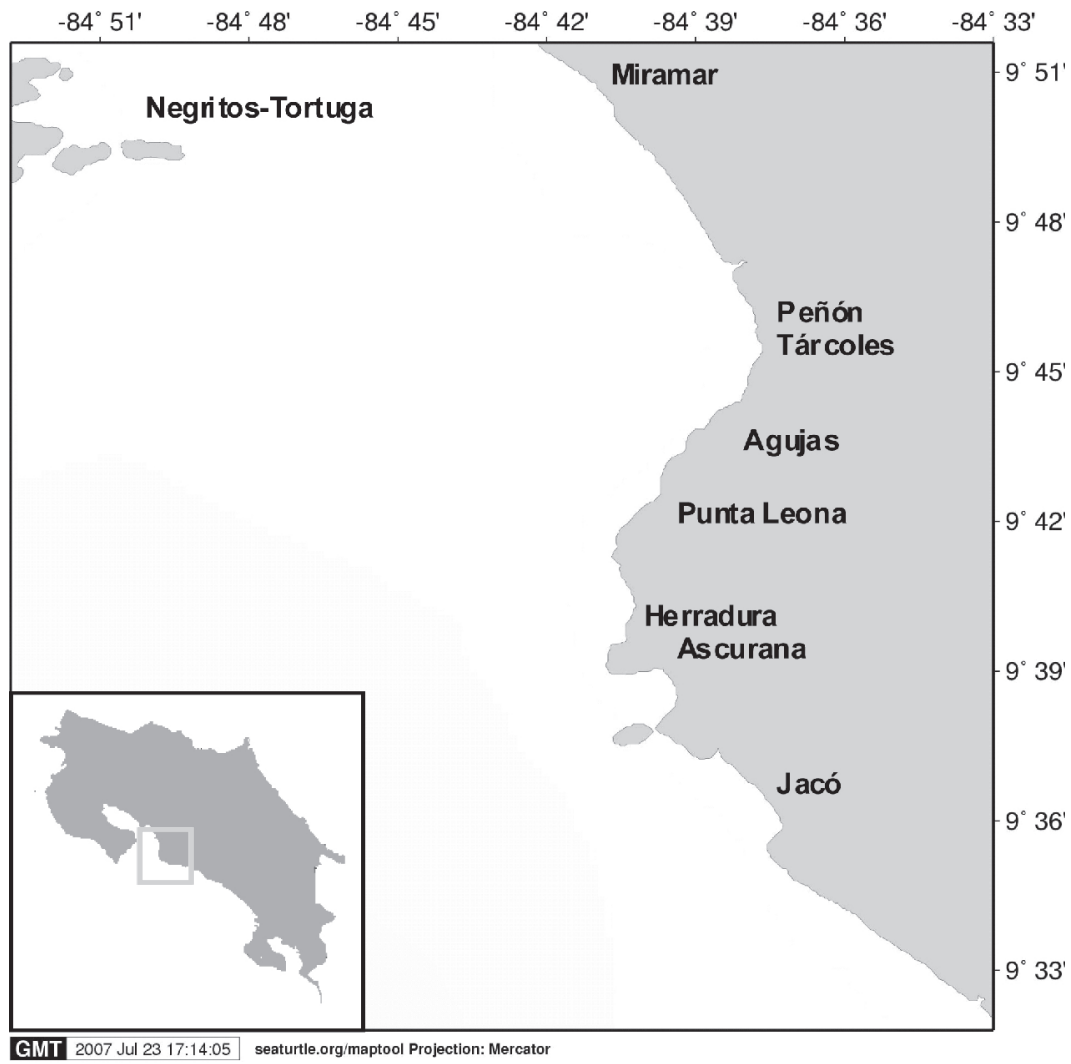

Localización geográfica de la comunidad costera de Tárcoles y principales zonas terrestres utilizadas por los pescadores como referencia en sus faenas. Pacífico de Costa Rica, 2006-2007. Location of the Tárcoles community and the main centers used by fishermen as reference for their fishing effort. Pacific coast of Costa Rica, 2006-2007.

zonas del Golfo de Nicoya, los pescadores de Tárcoles utilizan líneas de fondo o planeras, líneas rayeras y trasmallos para capturar pargos (Lutjanidae), róbalos (Centropomidae) y cabrillas (Serranidae), así como tiburones (Triakidae, Carcharhinidae, Sphyrnidae).

La línea de fondo consta de una línea madre (1-4 km de longitud), de la cual penden anzuelos tipo $\mathrm{J}\left(\mathrm{N}^{\mathrm{o}} 7 \mathrm{y}\right.$
8) cada 2 metros. Se utilizan de 700 a 2.000 anzuelos en cada faena de pesca, calando la línea durante la noche y permaneciendo en el agua por períodos de 2 a 12 horas hasta el virado, generalmente al amanecer. Como carnada utilizan sardinas, anguilas o peces pequeños obtenidos de la pesca semiindustrial de camarón o sardina. La línea rayera es muy similar a la línea de fondo, solo que se utiliza un 
menor número de anzuelos (75-150) de mayor tamaño (tipo $\mathrm{J}, \mathrm{N}^{\circ} 1$ ). A su vez, los trasmallos o "paños" son redes de $200 \mathrm{~m}$ de longitud, de $7.62 \mathrm{a}$ $12.7 \mathrm{~cm}$ ( 3 a 5 plg) de luz de malla. Las faenas con línea se realizan en zonas lodosas y rocosas como Tárcoles, Peñón, Punta Leona, Herradura y Jacó, mientras que la operación del trasmallo se realiza principalmente en las desembocaduras de ríos, como Tárcoles y Peñón (Figura 1). Se analizaron las capturas pesqueras de tiburones y rayas procedentes de dos fuentes de información: observación de descargas y observación a bordo. La información proveniente de cada fuente se analizó por separado.

En las observaciones de descarga se registró el arte utilizado y la zona de pesca (según el pescador), además de todos los tiburones y rayas látigo descargados. Con el fin de relacionar la abundancia de tiburones y rayas látigo con las zonas de pesca utilizadas por los pescadores, se realizó un análisis de correspondencia multivariado (CA) con el programa CANOCO, versión 4.5 para Windows (ter Braak y Smilauer, 2002). En los viajes de observación a bordo, se registró el sitio de pesca mediante un sistema de posicionamiento global (GPS), las horas de pesca efectiva, el número de anzuelos y el número total de individuos por especie de la captura comercial. Las principales especies capturadas fueron agrupadas en categorías según su comercialización (peces almacenados, peces descartados, tiburones y rayas látigo) y se estimó la contribución porcentual de cada grupo. La información proveniente de observaciones a bordo incluyó únicamente faenas realizadas con la línea de fondo.

Abundancia relativa: La abundancia relativa de tiburones fue expresada como la Captura por Unidad de Esfuerzo (CPUE), y se estimó a partir de los tiburones capturados por las líneas de fondo durante los viajes a bordo. Para estandarizar la fluctuación de la CPUE a lo largo del año, se consideró importante incluir en la función, el tiempo efectivo que los anzuelos estuvieron en el agua, el cual puede variar entre temporadas, entre pescadores, e inclusive, entre faenas de pesca. De esta manera se calculó la CPUE como el número de tiburones capturados por anzuelo por hora, mediante la siguiente fórmula:

$$
\begin{aligned}
& \text { CPUE }=(\mathrm{TT} / \mathrm{TA}) \\
& {[(\mathrm{HIV}-\mathrm{HIC}) / 2+(\mathrm{HFV}-\mathrm{HFC}) / 2]} \\
& \text { donde, }
\end{aligned}
$$

$\mathrm{TT}=$ Total de tiburones

$\mathrm{TA}=$ Total de Anzuelos

$\mathrm{HIV}=$ Hora Inicial Virado

$\mathrm{HIC}=$ Hora Inicial Calado

$\mathrm{HFV}=$ Hora Final Virado

$\mathrm{HFC}=$ Hora Final Calado

\section{RESULTADOS}

Entre febrero del 2006 y febrero del 2007, se observó la descarga 
Análisis de las capturas de tiburones y rayas en las pesquerías artesanales de Tárcoles, Pacífico Central de Costa Rica

de tiburones y rayas capturados por pescadores de Tárcoles. En total se recolectó información de 415 faenas de pesca artesanal, de las cuales 340 $(81.9 \%)$ fueron observaciones de descarga y $75(18.1 \%)$ se realizaron con un observador a bordo. La observación de descargas incluyó en su mayoría faenas realizadas con la línea de fondo $(90 \%)$, seguido por el trasmallo $(6 \%)$ y la línea rayera $(3 \%)$, además de algunas descargas aisladas, donde no fue posible determinar el arte de pesca utilizado (1\%). A su vez, las 75 observaciones a bordo fueron realizadas con líneas de fondo únicamente. Todas las faenas de pesca analizadas se realizaron en las zonas comprendidas entre las coordenadas 9 $9^{\circ} 50^{\prime}-9^{\circ} 30^{\prime} \mathrm{N}$ y $84^{\circ} 50^{\prime}$ $84^{\circ} 35^{\prime} \mathrm{W}$, en fondos lodosos y rocosos, con profundidades entre $10 \mathrm{y}$ poco más de 90 metros.

Observación de descargas: En total se observó la descarga de 340 faenas de pesca, en las cuales se analizaron 3.185 tiburones $(9$ especies diferentes) y 114 rayas látigo. El tiburón mamón enano (Triakidae: Mustelus henlei) fue la especie predominante en la captura total $(63.7 \%)$, seguido por el tiburón mamón común (Triakidae: Mustelus lunulatus) (16.7\%). Otras especies importantes fueron el tiburón picudo común (Carcharhinidae: Rhizoprionodon longurio) que representó el $6.9 \%$ y el tiburón martillo común (Sphyrnidae: Sphyrna lewini) con un $6.1 \%$. A su vez, la raya látigo (Dasyatidae: Dasyatis longa) y el ti- burón picudo fucsia (Carcharhinidae: Nasolamia velox) representaron un $3.5 \%$ y $2.6 \%$, respectivamente. También se descargaron ocasionalmente el tiburón toro (Carcharhinidae: Carcharhinus leucas), el tiburón punta negra (Carcharhinidae: Carcharhinus limbatus), el tiburón tigre (Carcharhinidae: Galeocerdo cuvier) y el tiburón zorro pelágico (Alopiidae: Alopias pelagicus), aportando entre todos el $0.5 \%$ de la captura total (Figura 2).

Con respecto a las capturas de tiburones y rayas látigo descargadas por zona de pesca, Herradura registró la mayor captura (2.517 individuos, $76.3 \%$ ), seguida por Jacó (202 individuos, 6.1\%), Punta Leona (175 individuos, 5.3\%), Tárcoles (114 individuos, 3.5\%) y el Peñón (95 individuos, $2.9 \%$ ). En otras zonas como Ascurana (72 individuos, 2.2\%), Negritos-Tortuga (49 individuos, 1.5\%), Agujas (39 individuos, 1.2\%), Miramar (16 individuos, $0.5 \%$ ) y "Otros" (20 individuos, $0.5 \%$ ) se registraron las menores capturas.

El análisis de correspondencia multivariado (CA), que asocia las zonas de pesca con las especies de tiburones y rayas látigo capturadas, revela una fuerte relación entre los tiburones mamones (Mustelus) y las zonas conocidas como Herradura y Ascurana. A su vez, las zonas del Peñón y Miramar se relacionan con el tiburón martillo común y la raya látigo. El tiburón picudo común y el tiburón picudo fucsia también se relacionaron, aunque en menor grado, 
Figura 2
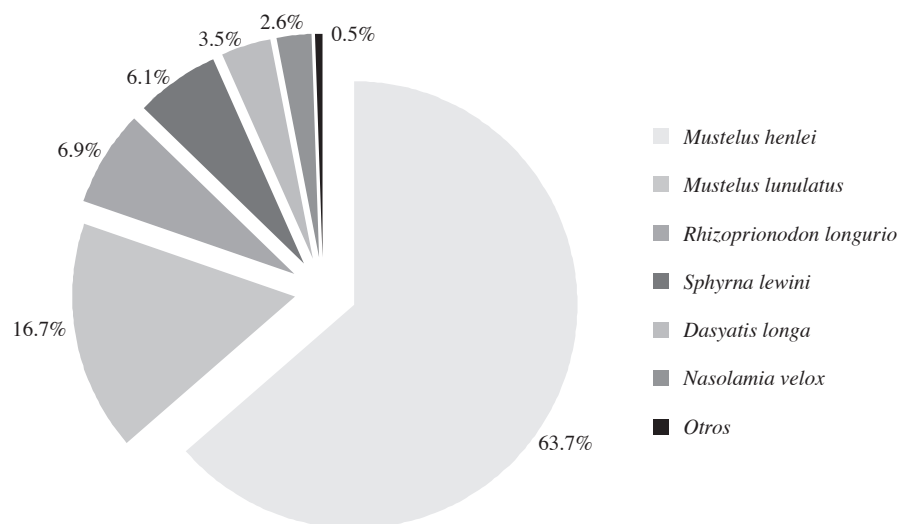

Aporte porcentual de las especies de tiburones y rayas látigo analizadas durante la observación de descargas. Tárcoles, Costa Rica, 2006-2007.

Percent contribution by species for sharks and longtail stingrays recorded during observations of dock side landings in Tárcoles, Costa Rica, 2006-2007.

con la zona de Punta Leona. Especies poco abundantes, como el tiburón zorro pelágico y el tiburón tigre, se relacionaron con Jacó y el Peñón, debido a que los pocos individuos analizados fueron capturados por los pescadores en las proximidades de estas zonas. Otras especies como el tiburón toro y el tiburón punta negra fueron las más alejadas del primer eje, sugiriendo que no se relacionaron con ninguna zona de pesca, posiblemente debido a su baja abundancia (Figura 3).

Observación a bordo: Durante el período de estudio, se analizaron las capturas de 75 viajes de pesca con línea de fondo en aguas con profundidades desde los 10 metros hasta poco más de 90 metros (Figura 4). En los viajes con un observador, las líneas de fondo capturaron 6.458 pe- ces, los cuales fueron divididos en 4 grupos: peces almacenados $(59.9 \%)$, peces descartados $(35.7 \%)$, tiburones $(3.5 \%)$ y rayas látigo $(0.9 \%)$. En total se analizaron 207 tiburones (5 especies diferentes) y 55 rayas látigo. Nuevamente $M$. henlei fue la especie más abundante $(50.4 \%)$, le siguieron $R$. longurio $(21.7 \%)$, D. longa (13.8\%), N. velox (5.8\%), S. lewini (5.8\%) y $M$. lunulatus (2.5\%) (Figura 5). Durante las observaciones a bordo, la zona de Herradura también sobresalió por ser el sitio de pesca donde se capturaron la mayoría de los tiburones (79\%), seguido por Tárcoles (9\%), Negritos-Tortuga (4\%), Jacó (3\%), Punta Leona (2\%) y "Otros" (3\%).

Abundancia relativa: La abundancia relativa promedio de tiburones, expresada como la Captura por Unidad de Esfuerzo (CPUE), fue 
Análisis de las capturas de tiburones y rayas en las pesquerías artesanales de Tárcoles, Pacífico Central de Costa Rica

Figura 3

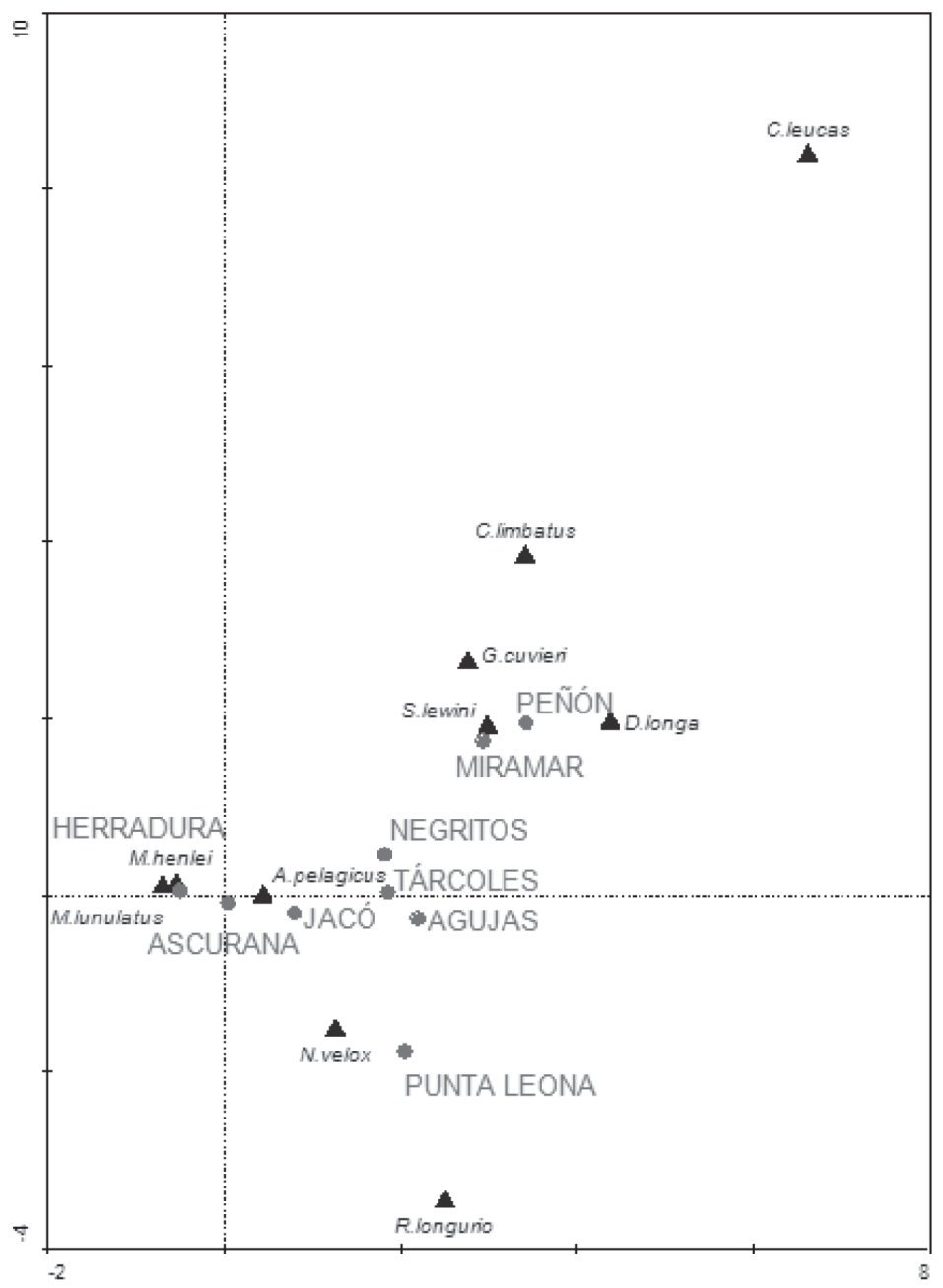

Análisis de correspondencia (CA) entre especies de tiburones y zonas de pesca. Observación de descargas. Tárcoles, Costa Rica, 2006-2007.

Canonical correspondence analysis between sharks species and fishing grounds. Dock side observations. Tárcoles, Costa Rica, 2006-2007. 


\section{Figura 4}

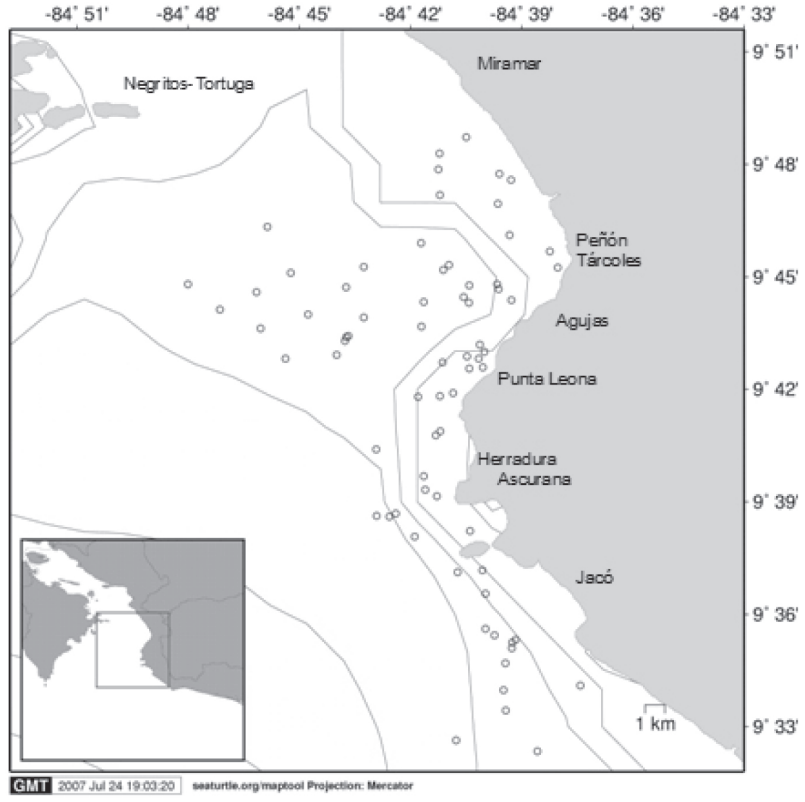

Localización geográfica de las zonas de pesca, donde se realizaron los 75 viajes a bordo de embarcaciones. Tárcoles, Costa Rica, 2006-2007.

Location of fishing grounds where on-board observations were carried out during 75 fishing outings. Tárcoles, Costa Rica, 2006-2007.

\section{Figura 5}

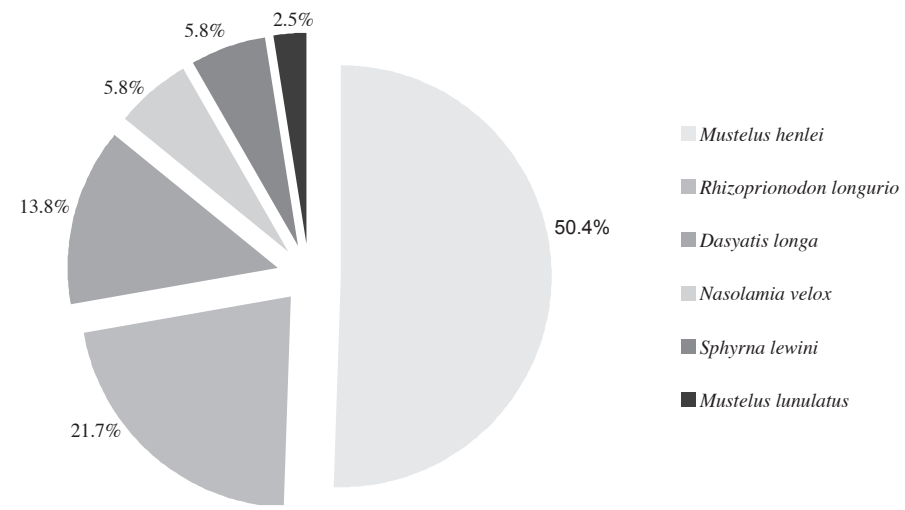

Aporte porcentual de las especies de tiburones y rayas látigo analizadas durante las observaciones a bordo. Tárcoles, Costa Rica, 2006-2007.

Percent contribution by species for sharks and longtail stingrays recorded during on-board observations. Tárcoles, Costa Rica, 2006-2007. 
Análisis de las capturas de tiburones y rayas en las pesquerías artesanales de Tárcoles, Pacífico Central de Costa Rica

variable durante el período de estudio. Los meses con mayores CPUE fueron setiembre y octubre, durante los cuales se capturaron en promedio 0.0016 tiburones por anzuelo por hora. Las menores CPUE se presentaron entre los meses de julio y agosto, durante los cuales se capturaron en promedio $3.36 \mathrm{E}-05$ tiburones por anzuelo por hora (Figura 6).

\section{DISCUSIÓN}

La línea de fondo fue el arte de pesca más utilizado por los pescadores de Tárcoles durante el período de estudio. El $81.9 \%$ de las descargas observadas y todos los viajes de observación a bordo se realizaron con este arte de pesca, cuyo uso es muy

Figura 6

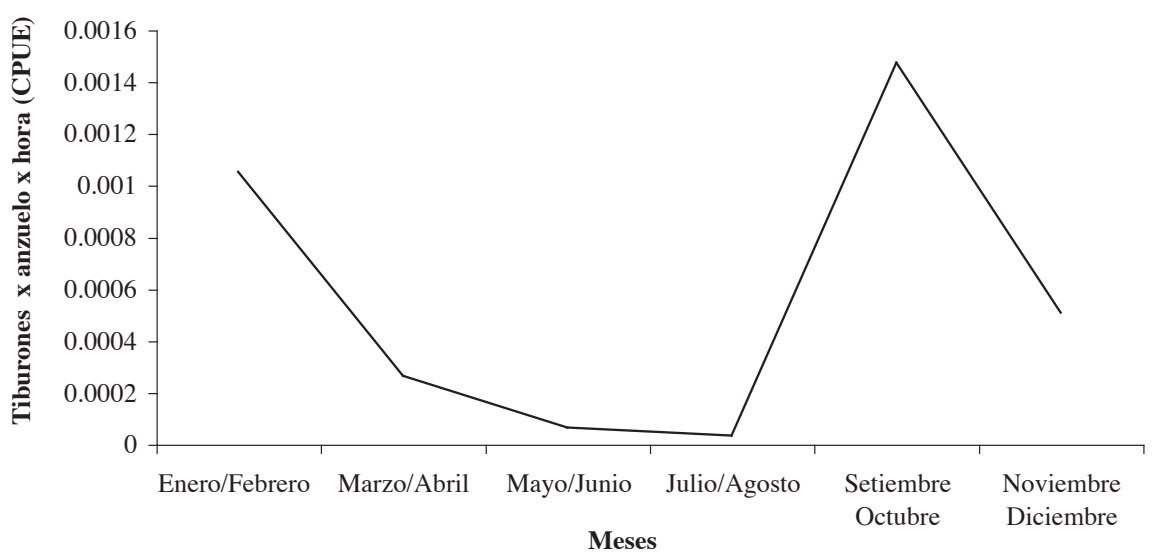

Promedios bimensuales de tiburones $\mathrm{x}$ anzuelo $\mathrm{x}$ hora, expresados como la Captura por Unidad de Esfuerzo (CPUE) de los tiburones capturados a bordo. Tárcoles, Costa Rica, 2006-2007.

Bimonthly averages for number of sharks per hook per hour, expressed as Catch Per Unit of Effort (CPUE) for shark on-board captures. Tárcoles, Costa Rica, 2006-2007.

común por la flota artesanal costarricense para la captura de pargo manchado (Lutjanus guttatus) y otros peces de fondo, incluidos tiburones y rayas (Villareal, 2001; Soto et al. 2009).

Las capturas pesqueras en las observaciones a bordo revelan que el $3.5 \%$ y el $0.9 \%$ de la captura total fueron tiburones y rayas látigo, respectivamente, un porcentaje muy similar al reportado para el Golfo de México, donde la captura de tiburones representa un $3.5 \%$ del total (Bonfil, 1997). En las faenas artesanales de los pescadores de Tárcoles 
sobresalen las especies de tiburones mamones (M. henlei y M. lunulatus) y picudos (R. longurio y $N$. velox), las cuales son especies costeras (Márquez et al. 2005). Sin embargo, también es posible observar especies oceánicas como el tiburón martillo común, el tiburón tigre y el tiburón toro, las cuales habitan el Golfo de Nicoya durante las etapas juveniles (Rojas et al. 2000).

Los tiburones mamones ( $M$. henlei y $M$. lunulatus) constituyeron el $80.4 \%$ de la captura total de elasmobranquios, esto mismo sucede en algunas áreas del Golfo de California, donde el género Mustelus representa el $80 \%$ de las capturas artesanales de elasmobranquios (Pérez et al. 2005), también en el Atlántico americano este género presenta importantes capturas pesqueras, en Estados Unidos (Conrath et al. 2002) y en Argentina (Chiaramonte \& Pettovello, 2000).

El análisis multivariado muestra las relaciones existentes entre las especies de elasmobranquios y las zonas de pesca. La relación más fuerte se da entre las zonas pesqueras identificadas como Herradura y Ascurana y los tiburones mamones (M. henlei y M. lunulatus). Existe también una fuerte relación entre el tiburón martillo común y la raya látigo con la zona del Peñón y Miramar, sitios de pesca ubicados en la parte más interna del Golfo de Nicoya, en aguas someras y de pendiente leve, frente a la boca del Río Tárcoles (Villareal, 2001). Esto se debe a que el tiburón martillo común es una especie que utiliza aguas costeras protegidas ricas en nutrientes, cercanas a las desembocaduras de ríos para parir sus crías (Klimley, 1987; Klimley et al. 1993; Anislado y Robinson, 2001; Duncan \& Holland, 2006). A diferencia del tiburón martillo, los ejemplares de la raya látigo capturados fueron en su mayoría adultos reproductivos, sobresaliendo las hembras grávidas que probablemente llegan a este sitio a parir sus crías, estas últimas poseen tallas pequeñas, por lo que no son susceptibles al arte de pesca (línea rayera). Es importante señalar que debido a su gran tamaño y peso, $D$. longa es la única especie de raya que se comercializa formalmente en Tárcoles. Esta especie aportó el 5\% de todos los elasmobranquios capturados, a diferencia de lo que sucede en el Golfo de California, donde se capturan más de 25 especies, representando el $63.4 \%$ de las capturas de elasmobranquios (Bizzarro et al. 2009).

La CPUE de tiburones por hora fue variable y estacional en el año, aumentando considerablemente en las faenas realizadas durante setiembre y octubre. Este aumento es influenciado por las capturas de M. henlei, sin discusión la especie más abundante y estacional en las pesquerías artesanales de Tárcoles. Al igual, Castillo et al. (1998) reportaron para el Golfo de México capturas variables y estacionales con las mayores CPUE de tiburones para el mes de octubre. De la misma forma, Smith et al. (2009) observaron diferencias temporales en 
Análisis de las capturas de tiburones y rayas en las pesquerías artesanales de Tárcoles, Pacífico Central de Costa Rica

las CPUE de tiburones en la pesquería artesanal de la costa oriental de Baja California, México. Inclusive estos mismos autores reportaron importantes picos estacionales de CPUE influenciados por tiburones del género Mustelus, debido a posibles agregaciones reproductivas o de alimentación. Se supone que con el aumento de las lluvias en estos meses arriban más nutrientes al sistema, producto de la sedimentación y escorrentía de los ríos, en especial del Río Tárcoles. Con el aumento de nutrientes, aumenta la disponibilidad de alimento, atrayendo a los consumidores secundarios y terciarios (peces herbívoros y carnívoros, respectivamente), los cuales a su vez atraen a depredadores tope, como los tiburones. Este comportamiento estacional ha sido reportado para otras especies del mismo género, tal es el caso de $M$. canis en Norteamérica y de M. lenticulatus en Nueva Zelanda (Francis y Mace, 1980). La zona de Herradura sobresalió como el sitio con la mayor abundancia y riqueza de tiburones, a pesar de que solo un $20 \%$ de las observaciones de descargas y un $35 \%$ de las observaciones a bordo se realizaron en esta zona. Herradura se ubica en las afueras del Golfo de Nicoya, donde los fondos son rocosos y profundos, con aguas más influenciadas por el mar abierto (Villareal, 2001); estas condiciones aparentemente propician la presencia estacional de los tiburones mamones (M. henlei y M. lunulatus).
La abundancia y estacionalidad de los tiburones en las zonas pesqueras cercanas a la comunidad de Tárcoles hacen que se promuevan estrategias de manejo para su conservación. Para los tiburones mamones (Mustelus) se propone un cierre técnico para las pesquerías en la zona de Herradura durante los meses con mayores capturas, como es el caso de enero-febrero y setiembre-octubre. Otra acción que se debe tomar urgentemente es restringir el uso del trasmallo y la línea rayera en la zona del Peñón. El tiburón martillo común y la raya látigo están fuertemente asociados a este sitio de pesca y a sus aguas protegidas ricas en nutrientes. Por último, ninguna estrategia de manejo para los tiburones y rayas látigo será efectiva, si se continúa ejerciendo la pesca de arrastre semiindustrial para camarón sin control en las cercanías a Tárcoles.

\section{AGRADECIMIENTOS}

Esta investigación fue posible gracias al apoyo incondicional de la Cooperativa de Pescadores Artesanales de Tárcoles (CoopeTárcoles R.L.). De nuevo gracias pescadores por su cooperación y por permitirnos analizar las capturas de tiburones y rayas de sus faenas artesanales. Además debemos agradecer a Conservación Internacional Centroamérica, Whitley Fund For Nature y Turtle Island Restoration Network, sin cuyo 
apoyo no hubiera sido posible realizar esta investigación.

\section{BIBLIOGRAFÍA}

Anislado, V. y Robinson, C. (2001). Edad y crecimiento del tiburón martillo Sphyrna lewini (Griffith y Smith, 1834) en el Pacífico Central de México. Ciencias: Biología de Sistemas Recursos Acuáticos. Cien. Mar., 27(4), 501-520.

Arauz, R., Cohen, Y., Ballestero, J., Bolaños, A. \& Pérez, M. (2004). Decline of shark populations in the Exclusive Economic Zone of Costa Rica. International Symposium. Quantitative Ecosystem Indicators for Fisheries Management. Paris, March 31-April 3.

Baum, J., Myers, R., Kehler, D., Worm, B., Harley, S. \& Doherty, P. (2003). Collapse and Conservation of Shark Populations in the Northwest Atlantic. Sci., 299 (5605), 389-392.

Bizzarro, J. J., Smith, W. D., Márquez-Farías, J. F., Tyminski, J. \& Hueter, R. E. (2009). Temporal variation in the artisanal elasmobranch fishery of Sonora, Mexico. Fish. Res., 97, 103-117.

Bonfil, R. (1997). Status of shark resources in the southern Gulf of Mexico and Caribbean: Implications for management. Fish. Res., 29, 101-117.

Castillo, J. L., Márquez, J. F., Rodríguez de la Cruz, M. C., Cortés, B. E. \& del Prado, A. (1998). The Mexican artisanal shark fishery in the Gulf of Mexico: towards a regulated fishery. Mar. Freshw. Res., 49, 611-620.

Chiaramonte, G. \& Pettovello, A. (2000). The biology of Mustelus schmitti in southern Patagonia, Argentina. J. Fish. Biol., 57, 930-942.

Conrath, C., Gelsleichter, J. \& Musick, J. (2002). Age and growth of the smooth dogfish (Mustelus canis) in the northwest Atlantic Ocean. Fish. Bull., 100, 674-682.

Coope SoliDar R.L. (2005). Tárcoles: una comunidad de pescadores artesanales en Costa Rica, aportes a la conservación de los recursos marino costeros del Golfo de Nicoya. San José, Costa Rica.

Duncan, K. \& Holland, K. (2006). Habitat use, growth rates and dispersal patterns of juvenile scalloped hammerhead sharks Sphyrna lewini in a nursery habitat. Mar. Ecol. Prog. Ser, 312, 211-221.

Francis, M. \& Mace, J. (1980). Reproductive biology of Mustelus lenticulatus from Kaikoura and Nelson. New Zealand. J. Mar. Freshwat. Res., 14(3), 303-311.

González, L., Herrera, A., Villalobos, L., Breton, Y., López, E., Breton, E., Houde, Y., Roy, D. y Benazera, C. (1993). Comunidades pesquero-artesanales en Costa Rica. Heredia, Costa Rica. Universidad Nacional de Costa Rica.

INRECOSMAR. (1999). Diagnóstico de la Pesca de Tiburón en Centro América. Proyecto colaborativo entre la Asociación Proambiente y el 
Análisis de las capturas de tiburones y rayas en las pesquerías artesanales de Tárcoles, Pacífico Central de Costa Rica

Programa PRADEPESCA, con el Apoyo Técnico del Instituto de Recursos Costeros y Marinos (INRECOSMAR). San José, Costa Rica.

Klimley, A. P. (1987). The determinants of sexual segregation in the scalloped hammerhead shark, Sphyrna lewini. Environ. Biol. Fish., 18(1), 27-40.

Klimley, A. P., Cabrera-Manzilla, I. y Castillo-Geniz, L. (1993). Descripción de los movimientos horizontales y verticales del tiburón martillo, Sphyrna lewini, del sur del Golfo de California de México. Cien. Mar., 19(1), 95-115.

Márquez, J. F., Corro, D. \& Castillo, J. F. (2005). Observations on the Biology of Pacific Sharpnose Shark (Rhizoprionodon longurio, Jordan and Gilbert, 1882), Captured in Souther Sinaloa, México. $J$. Northw. Atl. Fish. Sci., 35, 107-114.

Pérez, J. C., Sosa, O. \& Castillo, J. (2005). A New Eastern North Pacific Smoothhound Shark (Genus, Mustelus, Family Triakidae) from the Gulf of California. Copeia, 4 , 834-845.

Rojas, J. R., Campos, J., Segura, A., Mug, M., Campos, R. \& Rodrí- guez, O. (2000). Shark fisheries in Central America: a review and update. Uniciencia, 17, 49-56.

Smith, W. D., Bizzarro, J. J. y Cai1liet, G. M. (2009). La pesca artesanal de elasmobranquios en la costa oriental de Baja California, México: Características y consideraciones de manejo. Cien. Mar., 35(2), 209-236.

Soto, R., Mejía, F., Palacios, J. e Hiramatsu, K. (2009). Reproducción y crecimiento del pargo mancha Lutjanus guttatus (Pisces: Lutjanidae) en el Golfo de Nicoya, Costa Rica. Rev. Biol. Trop., 57(12), 125-131.

ter Braak, C. J. F. \& Smilauer, P. (2002). CANOCO reference manual and CanoDraw for Windows user's guide: software for canonical community ordination (version 4.5). Microcomputer Power, Ithaca, New York, USA.

Villareal, A. (2001). La composición de especies en la pesca artesanal con línea de fondo en la parte externa del Golfo de Nicoya, Costa Rica. Tesis de Licenciatura, Escuela de Ciencias Biológicas, Universidad Nacional. Heredia, Costa Rica. 